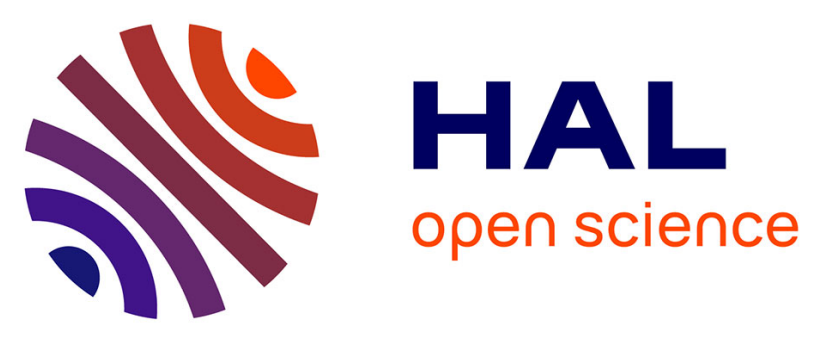

\title{
Dawn/dusk asymmetry of the Martian UltraViolet terminator observed through suprathermal electron depletions
}

Morgane Steckiewicz, P. Garnier, R. Lillis, D. Toublanc, François Leblanc, D. L. Mitchell, L. Andersson, Christian Mazelle

\section{To cite this version:}

Morgane Steckiewicz, P. Garnier, R. Lillis, D. Toublanc, François Leblanc, et al.. Dawn/dusk asymmetry of the Martian UltraViolet terminator observed through suprathermal electron depletions. Journal of Geophysical Research Space Physics, 2019, 124 (8), pp.7283-7300. 10.1029/2018JA026336 . insu02189085

\section{HAL Id: insu-02189085 \\ https://hal-insu.archives-ouvertes.fr/insu-02189085}

Submitted on 29 Mar 2021

HAL is a multi-disciplinary open access archive for the deposit and dissemination of scientific research documents, whether they are published or not. The documents may come from teaching and research institutions in France or abroad, or from public or private research centers.
L'archive ouverte pluridisciplinaire HAL, est destinée au dépôt et à la diffusion de documents scientifiques de niveau recherche, publiés ou non, émanant des établissements d'enseignement et de recherche français ou étrangers, des laboratoires publics ou privés. 


\section{JGR Space Physics}

\author{
RESEARCH ARTICLE \\ 10.1029/2018JA026336 \\ Key Points: \\ - The approximate position of the \\ UltraViolet terminator can be \\ determined thanks to suprathermal \\ electron depletions \\ - The UV terminator is at a mean \\ altitude of $123 \mathrm{~km}$ above the optical \\ terminator over one Martian year \\ - The UV terminator is higher on the \\ duskside than on the dawnside at \\ equinox and models predict an \\ inversion at aphelion and perihelion
}

Correspondence to:

M. Steckiewicz and P. Garnier, morganesteckiewicz.pro@gmail.com; pgarnier@irap.omp.eu

Citation:

Steckiewicz, M., Garnier, P., Lillis, R., Toublanc, D., Leblanc, F., Mitchell, D. L., et al. (2019). Dawn/dusk asymmetry of the Martian UltraViolet terminator observed through suprathermal electron depletions. Journal of Geophysical Research: Space Physics, 124, 7283-7300. https://doi. org/10.1029/2018JA026336

Received 28 NOV 2018 Accepted 13 JUL 2019

Accepted article online 18 JUL 2019 Published online 21 AUG 2019
(C)2019. American Geophysical Union. All Rights Reserved.

\section{Dawn/Dusk Asymmetry of the Martian UltraViolet Terminator Observed Through Suprathermal Electron Depletions}

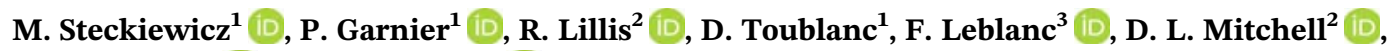 \\ L. Andersson ${ }^{4}$ (D), and C. Mazelle ${ }^{1}$ (iD) \\ ${ }^{1}$ IRAP, Université de Toulouse, CNRS, UPS, CNES, Toulouse, France, ${ }^{2}$ Space Sciences Laboratory, University of \\ California, Berkeley, CA, USA, ${ }^{3}$ LATMOS/IPSL, UPMC University Paris 06 Sorbonne Universités, UVSQ, CNRS, Paris, \\ France, ${ }^{4}$ Laboratory for Atmospheric and Space Physics, University of Colorado Boulder, Boulder, CO, USA
}

Abstract Suprathermal electron depletions are structures of the nightside ionosphere of Mars resulting from an equilibrium between electron loss and creation processes. Photoionization of oxygen and carbon dioxide by UV and EUV photons is the main ionization process of the Martian atmosphere. The observation of suprathermal electron depletions is strongly unexpected in the portion of the Martian environment where photoionization can occur. This region is delimited by the UltraViolet (UV) terminator, behind which no UV ionizing photons are detected. In this study suprathermal electron depletions are used to determine the position of the UV terminator thanks to MAVEN observations. The MAVEN spacecraft is now in its fourth year of data recording and has already covered more than one Martian year, a large range of latitude, local time, and solar zenith angle in the nightside down to 110-km altitude. This coverage enables us to determine the approximate position of the UV terminator over one Martian year. We then investigate the variation of its position on the dawnside and duskside and depending on seasons. Our results are compared with models of the Martian atmosphere and in situ data of the atmospheric composition which all highlight an asymmetry between the duskside and the dawnside at equinox. However, models show an inversion in the position of the dusk and the dawn UV terminator at perihelion and aphelion, which cannot yet be confirmed or disproved by the data.

\section{Introduction}

In this paper, we suggest a new method to determine the location of the ultraviolet (UV) terminator of the Martian atmosphere, based on the so-called suprathermal electron depletions (Steckiewicz et al., 2015). In this section, we will first describe the Martian ionosphere before we define the UV terminator and describe the nature of suprathermal electron depletions. Section 2 then details the MAVEN instruments and the suprathermal electron depletions data set used in our study. Section 3 describes the method used to identify the UV terminator location and shows its application to one Martian year. In section 4 we investigate the location of the UV terminator on dawnside and duskside. Section 5 provides a discussion on the evolution of the position of the UV terminator regarding seasons.

\subsection{The Martian Ionosphere}

The upper neutral atmosphere of Mars interacts directly with the solar wind particles and photons, modifying the plasma neighboring the planet and creating an ionosphere (Kivelson \& Russel, 1995). The process of ionization involving photons is called photoionization, and the process involving energetic particles is often called impact ionization. While photons mainly come from the Sun, the ionizing particles can come from the Sun, but also from the galaxy (cosmic rays), or from the ionosphere itself. The only requirement for the photons and energetic particles to ionize the neutral atmosphere is that their energy exceeds the ionization potential or binding energy of a neutral.

Atmospheric ionization is usually attributable to a mixture of these various sources, but one often dominates. The two predominant ionization processes at Mars are the following:

1. Photoionization: solar photons arriving on Mars mainly interact with $\mathrm{O}$ and $\mathrm{CO}_{2}$ by the following mechanisms: 


$$
\begin{gathered}
\mathrm{CO}_{2}+h \nu \rightarrow \mathrm{CO}_{2}^{++e^{-}} \\
\mathrm{O}+h \nu \rightarrow \mathrm{O}^{++e^{-}}
\end{gathered}
$$

These two reactions are both associated with an ionization threshold near $90 \mathrm{~nm}: 91.1 \mathrm{~nm}$ for oxygen and $89.9 \mathrm{~nm}$ for carbon dioxide. It corresponds to solar photons in the "extreme" ultraviolet (EUV) and UV wavelength ranges. Photoionization is the most important ionization process at Mars, in particular to create the dayside ionosphere.

2. Ionization by electron impact: it corresponds to the interaction of electrons coming from the solar wind or the magnetosheath with the atmospheric neutrals thus producing ions. This process is the dominant ionization process producing the nightside ionosphere (Fillingim et al., 2010; Lillis et al., 2011; Lillis \& Brain, 2013). Following is an example of such ionization by electronic impact with a neutral (M):

$$
e-+M \rightarrow M++e-+e-
$$

These ionization processes are however not the only important processes driving the dynamics and thermal state of the Martian ionosphere. A number of source processes, loss processes, and transport mechanisms shall be considered. At low altitudes (below $250 \mathrm{~km}$; see Sakai, 2016), the external suprathermal electrons are essentially absent, so that electrons are locally produced (primary or secondary) or transported and eventually degraded in energy. The electron energy spectrum keeps traces of the photoionization process with complex features below $15 \mathrm{eV}$ or due to the 30.4-nm HeII solar line (or by X-rays with Auger electrons close to $500 \mathrm{eV}$; Mitchell et al., 2000), but energy degradation or heating due to collisions (with neutrals or Coulomb collisions with electrons) will smooth these spectral features and steepen the slope of the spectrum (Cui et al., 2011).

\subsection{Definition of the UV Terminator}

The nightside, the dayside, and the terminator separating them are concepts which depend on the phenomenon, or on the process that is studied. The terminator is the boundary between the region where photons are received and the region where they are not. The optical terminator can then be defined as the boundary of the optical shadow of the planet, its altitude varying with the solar zenith angle (SZA). The SZA corresponds to the angle between the line linking the spacecraft to the center of Mars, and the line between the center of Mars and the center of the Sun. However, all the sunlight wavelengths do not reach the Martian surface. The neutral atmosphere of Mars interacts with the incoming photons, absorbing them more or less depending on their wavelengths, and hence modifying the location of the corresponding terminator. The UV terminator is especially interesting in the Martian case as the main ionization process of the neutral atmosphere is photoionization of $\mathrm{O}_{2}$ and $\mathrm{CO}_{2}$ by UV and EUV photons. Shane et al. (2016) for example used an arbitrary base altitude of $90 \mathrm{~km}$ instead of the surface of Mars, assuming that the light is attenuated by the atmosphere below this altitude, in order to quantify the deposition of photoelectron and solar wind electron populations on the nightside of Mars.

We will now describe the geometry of the nightside and terminator that will be used later in this paper. The optical shadow of Mars can be represented by a cylinder whose radius is the planet radius $R_{\text {Mars }}$ (left part of Figure 1). The nightside is in this case defined as follows, where $r$ is the radial distance from the center of Mars:

$$
\mathrm{SZA}>90+\operatorname{acos}\left(\frac{R_{\mathrm{Mars}}}{r}\right)
$$

A conic shadow, taking into account the distance of Mars from the Sun, could also have been considered. However, this would result in a cone whose angle is $8.5 \times 10^{-4 \circ}$. The difference between the cylindrical and the conic shadow at an altitude of $1,000 \mathrm{~km}$ would be of $\sim 65 \mathrm{~m}$, which is far below the resolution of our data. The cylindrical shadow is sufficiently accurate for our purpose. 

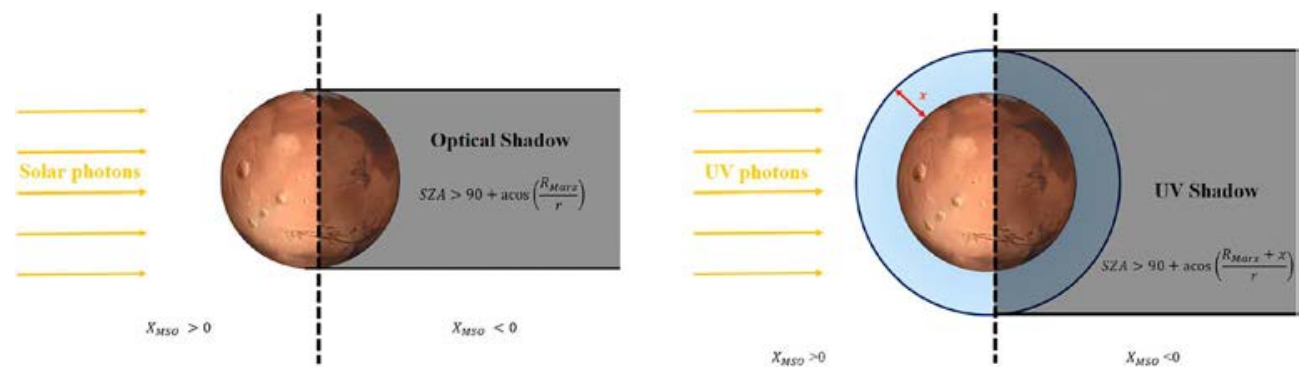

Figure 1. Definition of the nightside. On the left side is the optical shadow. On the right side is the UV shadow considered in this study.

As a first approximation we consider here that the UV shadow corresponds to the cylindrical shadow obtained with Mars surrounded by an atmospheric layer of $x \mathrm{~km}$ (right side of Figure 1), where all the EUV-UV photons are considered as absorbed. In this case, the nightside is defined as

$$
\mathrm{SZA}>90+\operatorname{acos}\left(\frac{R_{\mathrm{Mars}}+x}{r}\right)
$$

\subsection{Description of Suprathermal Electron Depletions}

In this paper, we will suggest a new method to determine the location of the UV terminator, based on suprathermal electron depletions. We will thus now remind the nature of these depletions.

Using measurements of the electron spectrometer onboard Mars Global Surveyor, Mitchell et al. (2001) first observed that the nightside ionosphere was punctuated by abrupt drops of the instrumental count rate, by up to 3 orders of magnitude to near background levels across all energies, hence calling them "plasma voids." These structures, renamed "suprathermal electron depletions" (hereinafter called electron depletions) by Steckiewicz et al. (2015) after new observations obtained with the MAVEN spacecraft, are the result of an equilibrium between electron loss and creation processes. Their observation is strongly linked to the magnetic topology of Mars. Mars indeed does not possess a global dynamo magnetic field at present time but possesses crustal sources, remnants from an ancient planetary magnetic field. The crustal magnetic sources, mainly located in the southern hemisphere, can be considered as small dipoles located under the Martian crust (Acuña et al., 1998). They form closed magnetic field loops that can extend up to thousands of kilometers above the surface.

In the Martian environment, suprathermal electrons are typically magnetized (with the gyrocenters of their helical motion constrained to follow the magnetic field lines). Closed magnetic field lines with both ends intersecting the dayside ionosphere fill with photoelectrons and are essentially isolated from solar wind electrons. The trapped electrons bounce back and forth between the two mirror points and the whole electron population undergoes absorption by the collisional neutral atmosphere (among which $\mathrm{CO}_{2}$ is the major species) if the mirror points are low enough (due to the convergence of the magnetic field lines as in the cusps). However, as photoelectrons are continuously created in the dayside ionosphere, no depletions in the suprathermal electron population are observed.

When closed crustal magnetic field loops rotate toward the nightside with the planet rotation, there is a transition period when one foot of the closed magnetic field loop is on the dayside and the other is on the nightside. This configuration can induce precipitations of photoelectrons in the nightside (Xu et al., 2017). When these closed field lines continue their rotation and found their both ends in the nightside, absorption by the collisional atmosphere continues, whereas fewer electrons are locally produced (mostly by electron impact ionization). The electron population inside closed crustal magnetic field loops is then thermalized via collisions with the neutral atmosphere, leaving only the thermal population and a remaining suprathermal electron population peaked at 6-7 eV, due to the shape of the collisional cross section with $\mathrm{CO}_{2}($ Steckiewicz et al., 2015). Electrons coming from other sources, such as horizontal transport of photoelectrons from dayside to nightside or solar wind plasma traveling up the magnetotail, are routed along the outermost magnetic field lines of the closed magnetic field loops toward the neutral atmosphere. They hence are not 


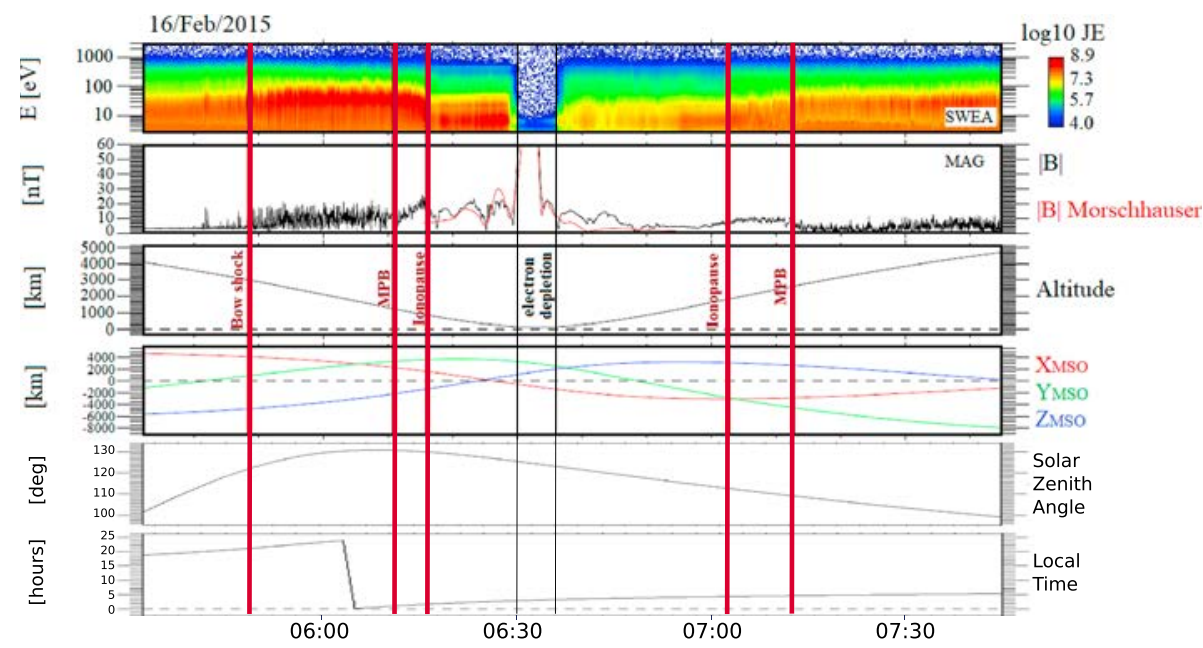

Figure 2. Example of a MAVEN passage in the plasma environment of Mars with a periapsis on the nightside. (panel 1) SWEA energy-time spectrogram of omnidirectional electron energy flux. (panel 2) Magnetic field intensity (measured by MAG in black and calculated from the crustal field model of Morschhauser et al. (2014) in red) versus time. (panel 3) Areocentric altitude versus time. (panel 4) Coordinates of the spacecraft in the MSO frame. The vertical lines highlight the main boundaries of the Martian environment. (panel 5) Solar zenith angle of the MAVEN spacecraft. (panel 6) Local time of the MAVEN spacecraft.

able to penetrate inside these loops to repopulate them: the inside of closed magnetic field loops forms suprathermal electron depletions.

However, the electron motion is governed by electric and magnetic fields only above the electron exobase. Below it, electron motion is dominated by collisions rather than by the magnetic field. All electrons in this region are then subjected to collisions, not only those travelling along closed magnetic field lines. The thermalization of the suprathermal electron population no longer depends directly on the geographical distribution of the crustal magnetic sources. The distribution of electron depletions in this region is then more homogeneous than at higher altitudes (Steckiewicz et al., 2016). The electron exobase is generally found around 170-km altitude (Lillis et al., 2008; Mantas \& Hanson, 1979; Steckiewicz et al., 2015; Xu et al., 2016).

An example of electron depletion observed by MAVEN on 16 February 2015 is plotted in Figure 2. We can see that hardly any electrons can be observed between 06:30 UT and 06:33 UT, except a faint line at $6 \mathrm{eV}$, due to absorption by atmospheric $\mathrm{CO}_{2}$. This case reveals the presence of strong crustal magnetic fields precisely at the location of the electron depletion: the closed fields prevent from refilling the depletion via external plasma sources.

\section{Instrumentation and Suprathermal Electron Depletions Data Set Used}

We will now describe the MAVEN instruments that will be used in this study, how these instruments allow to observe the UV terminator, and finally describe the data set of suprathermal electron depletions that will be the basis of our new method to determine the UV terminator.

\subsection{MAVEN Instruments}

After its launch in November 2013, the MAVEN spacecraft spent 10 months in its interplanetary journey and finally reached Mars on 21 September 2014 (Jakosky et al., 2015). Once the science mapping orbit has been reached, the Science phase began on 16 November 2014. The science mapping orbit is a highly elliptical orbit, with a nominal periapsis at $150 \mathrm{~km}$, and an apoapsis of $\sim 6,220 \mathrm{~km}$, which means a period of $\sim 4.5 \mathrm{hr}$. The periapsis is periodically lowered down to $125 \mathrm{~km}$ for five-day periods known as "deep-dips" (Bougher et al., 2015). The orbital inclination of $75^{\circ}$ has been chosen together with the apoapsis altitude and the orbital period to provide an appropriate precession rate of the orbit in both local time and latitude.

The MAVEN spacecraft carries onboard a complete suite of plasma and field instruments, plus an ultraviolet spectrometer and a neutral spectrometer. In this study we only focus on three of these instruments: 
1. SWEA (Solar Wind Electron Analyzer): the SWEA experiment (Mitchell et al., 2016) measures the energy and angular distributions of solar wind and magnetosheath electrons and ionospheric photoelectrons. Its energy range is 3 to $4,600 \mathrm{eV}$ and its energy resolution is $\frac{\delta E}{E}=17 \%$. SWEA has a 2 -s measurements cadence.

2. NGIMS (Neutral Gas and Ion Mass Spectrometer): the NGIMS experiment (Mahaffy et al., 2015) makes in situ measurements of the neutral composition, isotopic ratios, and scale height temperature of the major gas species and thermal ions in the Martian upper atmosphere.

3. MAG: the MAGnetic field investigation (Connerney et al., 2015) consists of dual-triaxial magnetometers located at the end of "boomlets" on both ends of the solar panel arrays of the spacecraft. MAG samples the magnetic field at 32 vector observations per second.

\subsection{The UV Terminator Observed by MAVEN Instruments}

The UV terminator can be observed thanks to both electron spectrometers (as SWEA on MAVEN) and Langmuir probes (as LPW on MAVEN). Depending on the plasma in which it is embedded, a Langmuir probe measures essentially two currents in the negative potential regime: the photoelectron current (induced by the removal of electrons at the surface of the probe by photoionization) and the ion current (induced by the impact of the ambient ions on the probe). At low plasma density, such as at high altitudes, the photoelectron current dominates, whereas at high plasma densities, such as in the ionosphere, the ion current dominates. Concerning the electron spectrometer, it directly measures photoelectrons resulting from the photoionization process (both natural and induced photoelectrons).

Figure 3 shows a MAVEN periapsis observed by the SWEA (Mitchell et al., 2016) and LPW (Andersson et al., 2015) instruments. On the first panel is plotted the omnidirectional electron energy-time spectrogram recorded by SWEA, on the second panel is the current/voltage spectrogram recorded by LPW, and on the third and fourth panel are the altitude and the position of the spacecraft in the Martian environment (see section 2).

We note that all the ephemerides used in this paper are expressed in the MSO (Mars-centric Solar Orbital) coordinates defined as follows: the origin is the center of Mars, the $x$ axis points from the center of Mars to the Sun, $y$ axis points opposite to Mars' orbital angular velocity, and $z$ completes the right-handed set so that the frame rotates slowly as Mars orbits the Sun. If no precision is given, the areocentric altitude is considered (the reference frame is a sphere with Mars' volumetric mean radius of 3,389.51 km).

Two crossings of the UV terminator are present in Figure 3. The first one is located at 19:30 UT and can be observed both on the SWEA spectrogram and on the LPW I-V spectrogram (red vertical line). It is located at high enough altitudes so that the dominant current measured by LPW is the photoelectron current. As the spacecraft crosses the UV terminator from dayside to nightside, no more photoelectrons are produced. The photoelectron flux measured by SWEA below $20 \mathrm{eV}$ and the current measured by LPW (at negative potentials; i.e., below the floating potential) decrease suddenly, showing a clear boundary. As the spacecraft passes its periapsis, $x_{\mathrm{MSO}}$ reverses $\left(\mathrm{SZA}=90^{\circ}\right)$ at $\sim 20: 20 \mathrm{UT}$, so that the UV terminator should be crossed close to that point. However, as the spacecraft is at low altitudes, the ion current dominates so that the identification of the UV terminator is more difficult with LPW. The identification in the SWEA data is also not easier at first glance due to the presence of a depletion in the electron flux induced by a significant change in the spacecraft potential (see section 2.3).

\subsection{The Suprathermal Electron Depletions Data Set}

In order to obtain a catalog of electron depletions observed by MAVEN we applied from November 2014 to March 2017 the criterion described in Steckiewicz et al. $(2015,2016)$ to detect automatically electron depletions in MAVEN data.

Due to the precession of the MAVEN orbit, electron depletions have only been detected during five specific periods, gathered in Table 1. These periods correspond roughly to the passage of the spacecraft at low enough altitudes in the nightside of the planet $\left(\mathrm{SZA}>90^{\circ}\right)$.

Each period of detection of electron depletions sampled a specific range of latitudes and can be related to a Martian season (seasons are due to the Mars inclination on its orbital plane is $25.19^{\circ}$, which is comparable to the one of the Earth: $23.44^{\circ}$ ): 


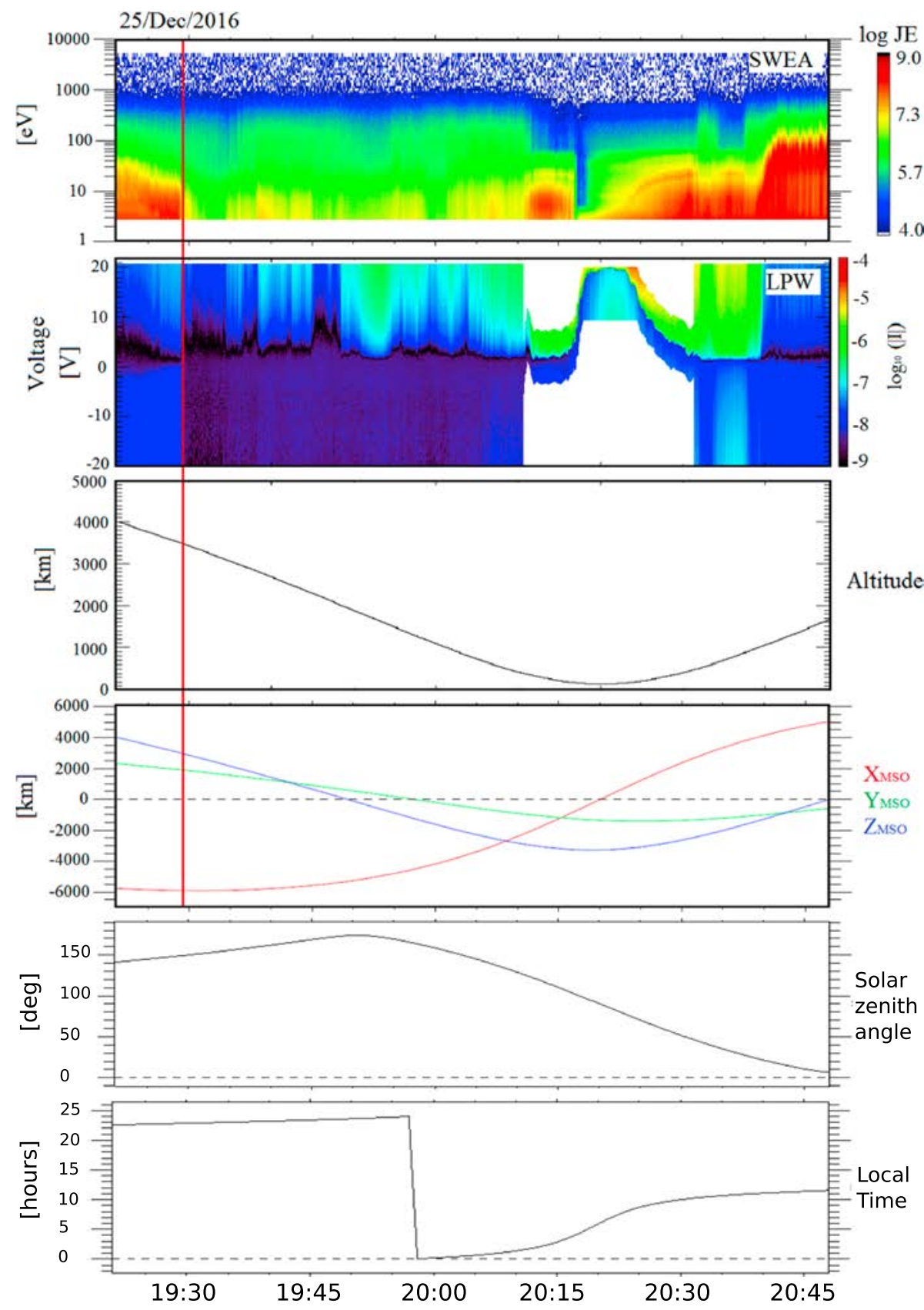

Figure 3. Observation of the UV terminator with the LPW and SWEA instruments onboard MAVEN. (panel 1) SWEA energy-time spectrogram of omnidirectional electron energy flux (ENGY mode). (panel 2) Current-voltage spectrogram by LPW. (panel 3) Areocentric altitude of the MAVEN spacecraft. (panel 4) Position of the MAVEN spacecraft in the MSO coordinates. (panel 5) Solar zenith angle of the MAVEN spacecraft. (panel 6) Local time of the MAVEN spacecraft.

1. First period: Winter of the northern hemisphere (Mars perihelion)

2. Second period: Spring of the northern hemisphere (equinox)

3. Third period: Summer of the northern hemisphere (Mars aphelion)

4. Fourth period: Autumn of the northern hemisphere (equinox)

5. Fifth period: Winter of the northern hemisphere (Mars perihelion).

One issue of the criterion used to automatically detect electron depletions is that it also detects spacecraft charging events. Usually, the spacecraft potential in the nightside ionosphere is approximately of $-2 \mathrm{~V}$. 
Table 1

Characteristics of the Five Periods During Which Electron Depletions Have Been Detected in the MAVEN Data

\begin{tabular}{|c|c|c|c|c|c|}
\hline & Time period & Median latitude $\left({ }^{\circ} \mathrm{N}\right)$ & Solar longitude $\left(^{\circ}\right)$ & Distance to the Sun (AU) & Number of depletions \\
\hline First period & $11 / 2014-02 / 2015$ & $63^{\circ}$ & $228-297$ & $1.42-1.38$ & 66,540 \\
\hline Second period & $06 / 2015-09 / 2015$ & $-70^{\circ}$ & $0-49$ & $1.50-1.62$ & 87,830 \\
\hline Third period & $12 / 2015-03 / 2016$ & $26^{\circ}$ & $77-128$ & $1.66-1.62$ & 49,966 \\
\hline Fourth period & $06 / 2016-09 / 2016$ & $-21^{\circ}$ & $160-235$ & $1.55-1.40$ & 150,122 \\
\hline Fifth period & $12 / 2016-03 / 2017$ & $-13^{\circ}$ & $272-338$ & $1.38-1.46$ & 88,800 \\
\hline
\end{tabular}

This implies a little modification in the energies detected, which are reduced by the same amount. Such small potentials have no significant impact on the criterion results. However, some strong spacecraft charging events can bring the spacecraft potential to a dozen of volts. An example of such event is presented in Figure 4. The charging event can be observed between 18:50 and 18:54 UT. A typical modification of the electron spectrum can be observed: two distinct lines appear around $10 \mathrm{eV}$, corresponding to the two photoelectron lines initially at 21-24 and $27 \mathrm{eV}$ (Mitchell et al., 2001)) that become shifted in energy due to charging. The electron flux detected at $6 \mathrm{eV}$ during this kind of event is then much lower than the mean electron flux calculated over $1 \mathrm{hr}$ and an electron depletion can be detected, whereas no electron depletions is effectively observed. A few cases have been found during the time period under study and have been removed by hand, as much as possible. Some of these cases are indeed located on the dayside so that they do not have any incidence on the detection of electron depletions. However, in December 2016/January 2017 a lot of spacecraft charging events have been observed near the terminator, impacting the detection of electron depletions. As our criterion detects the same way electron depletions and spacecraft charging events, electron depletions detected by our criterion may appear wider in time than real when spacecraft charging occur close to the depletions. An example of such "overflow" can be observed in Figure 4. The black vertical lines represent the boundaries of the electron depletions detected by our criterion. Two electron depletions have been detected in the nightside and close to the terminator (SZA $=90^{\circ}$ at $\sim 18: 51 \mathrm{UT}$ ). As the first electron depletion is roughly well delimited, the second one extends until 18:51:32 UT while it should stop at $~ 18: 50$ UT. The spacecraft charging event is detected in this case as an electron depletion. As there is no boundary between both structures, from the point of view of the criterion, it results in a very large electron depletion, which spreads toward the dayside.

Due to this specific configuration, a lot of electron depletions detected in December 2016/January 2017 appear wider than they truly are. We removed from our data set all the suprathermal depletions that occurred during a strong charging event, based on the composite spacecraft potential value provided by the MAVEN instruments (SWEA/LPW/STATIC), as well as those where the spacecraft potential was undefined. This removes almost half of the depletions detected during the December 2016/January 2017 (see section 4.2 for the consequences on the UV terminator study).

\section{Determination of the Position of the UV Terminator Using Suprathermal Electron Depletions}

Electron depletions are not restricted to the nightside defined as the optical shadow. Hall et al. (2016) observed that these structures are also observed in the illuminated induced magnetosphere, which is the region of space inside the magnetic pileup boundary and outside the optical shadow of the planet. Electron depletions are indeed the result of a balance between electron loss and production processes. In first approximation (neglecting transport and local production of electrons by electronic impact), electron depletions can be observed when no more photoelectrons are created in the ionosphere. As photoelectrons are mainly produced by photoionization of the neutral atmosphere by EUV and UV photons, the limit of detection of electron depletions corresponds in first approximation to the UV terminator.

\subsection{Methodology}

Based on equation (5) describing the UV terminator geometry, we can find its approximate location by looking for the thickness of the atmosphere $x$ which best fits the distribution of electron depletions. 


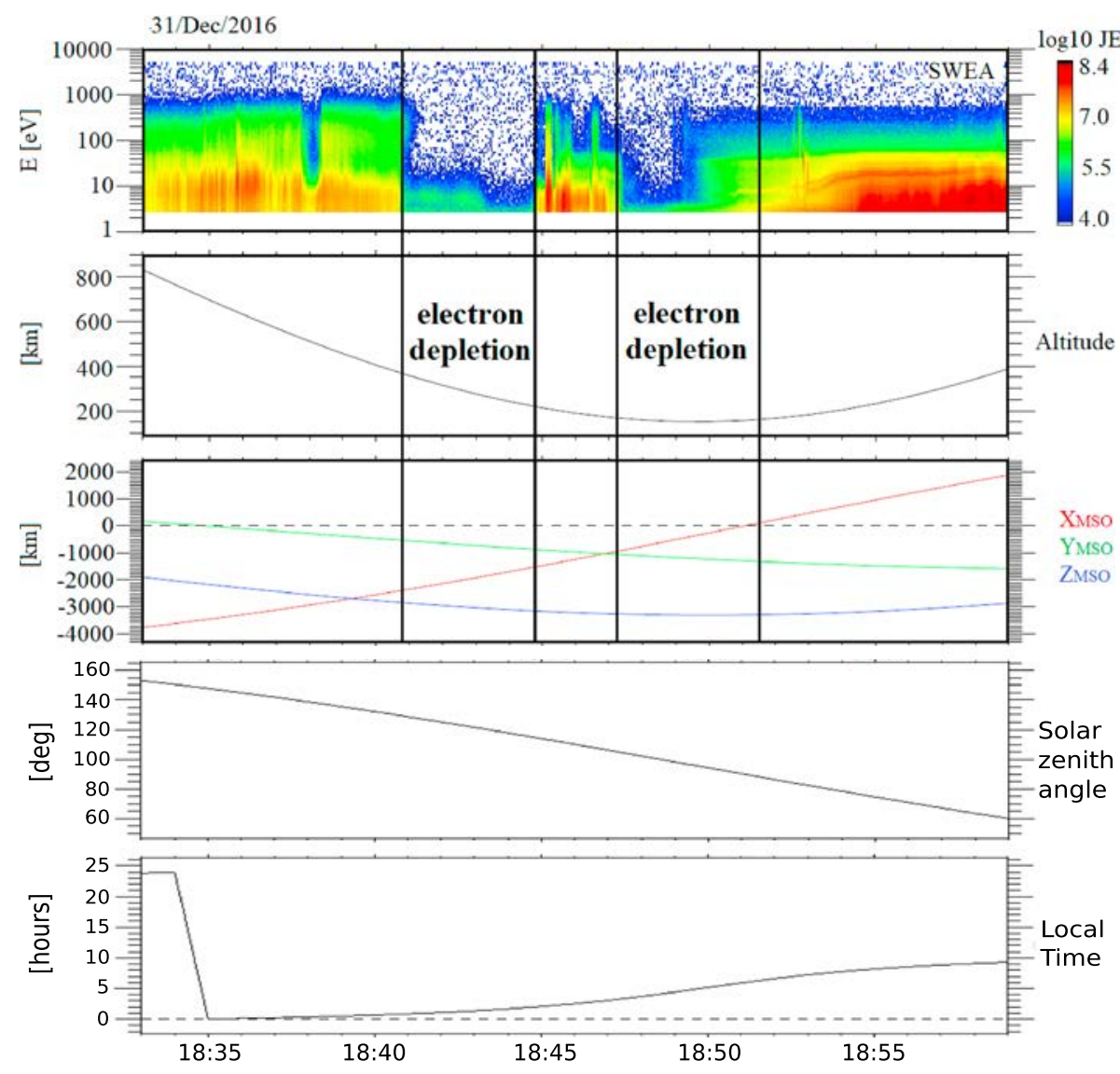

Figure 4. Example of overflow of an electron depletion detected by our criterion due to spacecraft charging. (panel 1) SWEA energy-time spectrogram of omnidirectional electron energy flux (ENGY mode). (panel 2) Altitude of the MAVEN spacecraft. (panel 3) Position of the MAVEN spacecraft in the MSO coordinates. The black vertical lines highlight the boundaries of the electron depletions detected with our criterion. (panel 4) Solar zenith angle of the MAVEN spacecraft. (panel 5) Local time of the MAVEN spacecraft.

In order to find the best fit between our data and the modeled UV terminator we binned electron depletion observations per MAVEN passage in altitude (10-km bins) and SZA ( $1^{\circ}$ bins) to obtain a statistical picture of their distribution. We then determined for each SZA the highest altitude having a percentage of electron depletions greater than $5 \%$. This threshold is arbitrary but enables a clear observation of the boundary under study. We finally use a nonlinear least mean square algorithm to determine the thickness $x$ of the atmosphere which best fits this $5 \%$ line (see equation (5)). Several tests performed show that using thresholds of, respectively, $1 \%$ or $10 \%$ instead of $5 \%$ lead to a small change of the resulting thickness $x$, respectively, by about 2 and $5 \mathrm{~km}$, which is small compared with the variations discussed below. As this study needs a good precision in altitude, we use the areodetic altitude, which takes into account the flattening at the poles $\left(R_{\text {equator }}=3,396 \mathrm{~km}\right.$ and $\left.R_{\text {pole }}=3,376 \mathrm{~km}\right)$.

\subsection{Application to One Martian Year}

At first, we chose to use all data obtained during the first four periods, which cover the four Martian seasons. Figure 5 shows percentages of electron depletions observations per MAVEN passage binned in altitude and SZA. The nonhomogeneity of the distribution in the nightside defined as SZA $>90^{\circ}$ catches the eyes. No electron depletion can be observed near SZA $=90^{\circ}$, except at very low altitudes (despite a good spatial coverage by the spacecraft). Generally, the lower the altitude is, the closer to $\mathrm{SZA}=90^{\circ}$,electron depletions can be observed.

We observed a clear boundary between the regions where electron depletions are observed and where they are not, which does not correspond to the optical terminator (dark blue line). The light blue line 


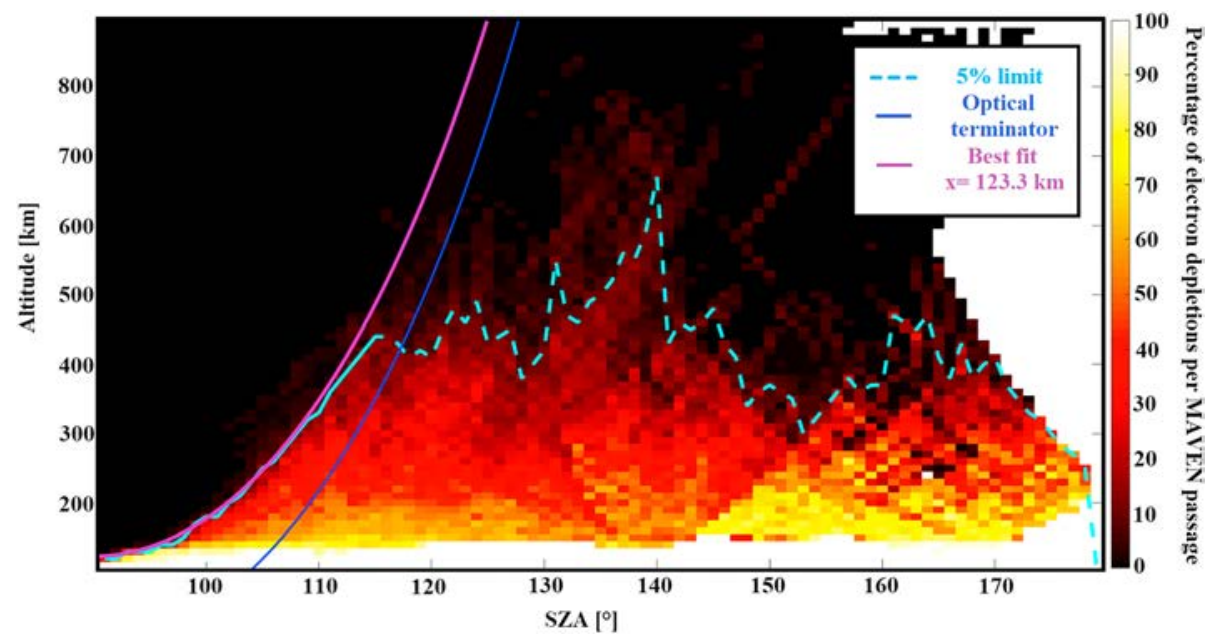

Figure 5. Distribution of the percentage of electron depletions per MAVEN orbit in SZA versus altitude. Data from November 2014 to March 2017 have been taken into account. The areodetic altitude has been used. The dashed blue line is the limit of 5\% electron depletions detected per MAVEN passage, the plain blue line corresponds to the data used for the fit, and the purple line is the best fit obtained. The dark blue line corresponds to the optical terminator.

corresponds to the limit of $5 \%$ electron depletions, and was chosen based on both good orbit coverage and on large enough percentage values in the bins. Only the low-altitude part of this $5 \%$ limit is used (i.e., the plain light blue line), since the use of electron depletions to observe the UV terminator is more relevant at low altitudes than at high altitudes due to the variable local conditions at high altitudes (local magnetic topology due to crustal fields, transport/precipitation of external electrons). The upper bound of the fitting part of the limit was chosen by eye for each plot. In this case, the best value found is $x=123.3$ $\mathrm{km}$ (purple line), with a coefficient of determination of $R^{2}=0.9815$ and a $95 \%$ confidence interval of $(119.7,126.9 \mathrm{~km})$.

We can see that the UV terminator fits well the data at low altitudes, but the consistency decreases above 330-km altitude. This shifting can be due to photoelectrons precipitating into the nightside on closed field lines that straddle the terminator (Xu et al., 2017), preventing the formation of electron depletions. This day-night magnetic connectivity hence provides a source of plasma and energy to the nightside. From a preliminary examination of hundreds of orbits, $\mathrm{Xu}$ et al. (2017) found that such nightside precipitations of photoelectrons are quite common.

\subsection{Comparison With an Atmospheric Model}

Figure 6 shows the transmission rate of ionizing EUV photons as a function of the radial distance and SZA as well as the $10 \%$ transmission threshold distance and altitude. Temperature-independent photoabsorption cross sections for $\mathrm{N}_{2}, \mathrm{CO}, \mathrm{Ar}$, and $\mathrm{O}$ were taken from the PHIDRATES database (https://phidrates.space. swri.edu/; Huebner \& Mukherjee, 2015), while fully temperature-dependent cross sections for $\mathrm{CO}_{2}$ (the dominant gas) were taken from Venot et al. (2018). A convolution of a typical solar moderate EUV spectrum measured by the MAVEN Extreme Ultraviolet Monitor (Eparvier et al., 2015; Thiemann et al., 2017) with the transmission rate at the various wavelengths between 10 and $89 \mathrm{~nm}$ has been made, $89 \mathrm{~nm}$ being the longest wavelength which can still ionize $\mathrm{CO}_{2}$. The neutral densities were taken taken from the Mars Climate Database version 5.2 (MCD; http://www-mars.lmd.jussieu.fr/mcd_python/; González-Galindo et al., 2009), with two extreme conditions of the Martian atmosphere (perihelion at dawn and aphelion at dusk). The MCD is a database of atmospheric simulation results compiled from the LMD Mars Global Climate Model (MGCM) simulations of the Martian atmosphere (Forget et al., 1999). This model computes in 3D the atmospheric circulation and climate of Mars taking into account the water cycle, radiative transfer, and the $\mathrm{CO}_{2}$ ice condensation and sublimation, among others.

If one considers equation (5) as an equality for a specific transmission threshold value, one can derive the UV terminator altitude, $x$, fitting the best the right panel curves of Figure 6. Considering a 10\% transmission 

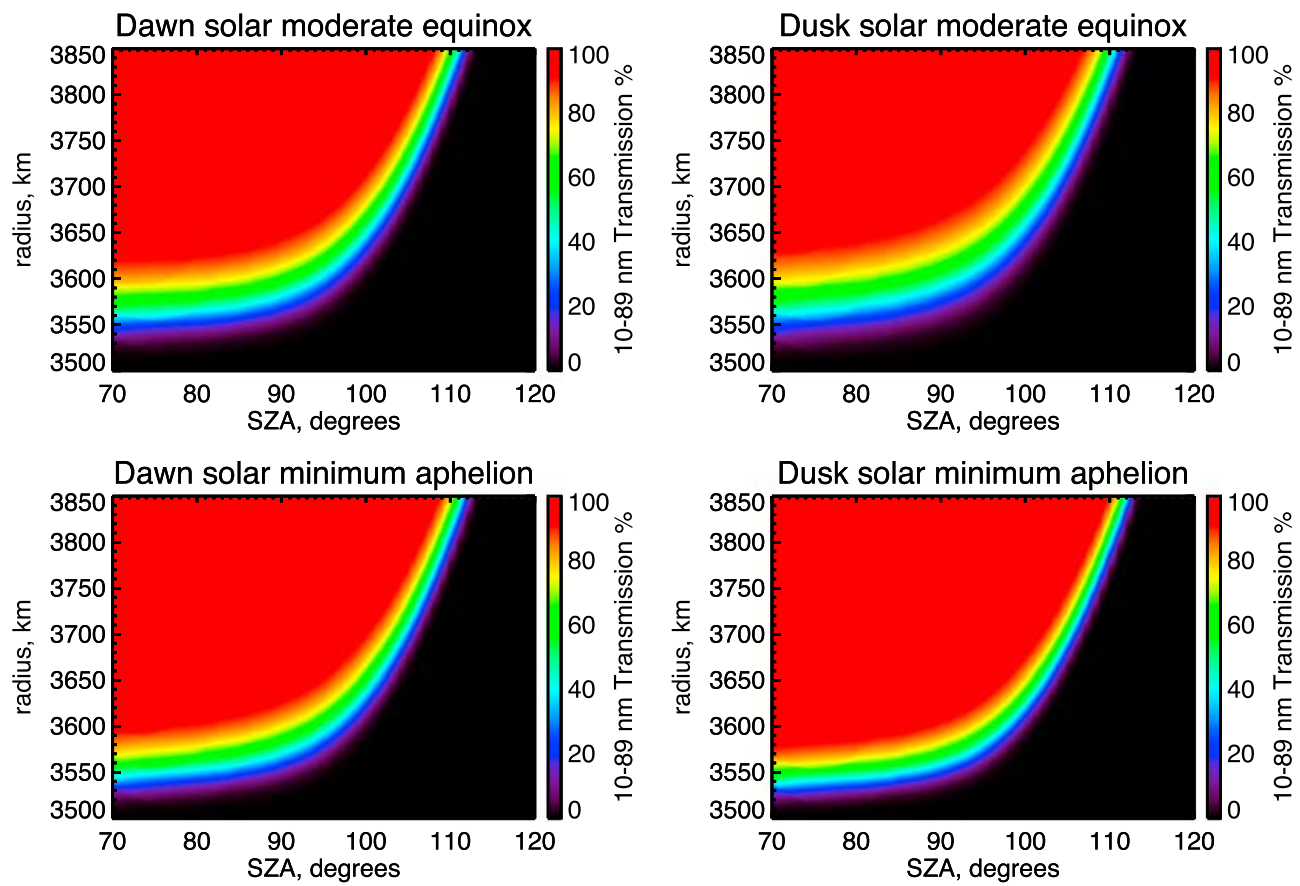

Dawn solar maximum perihelion
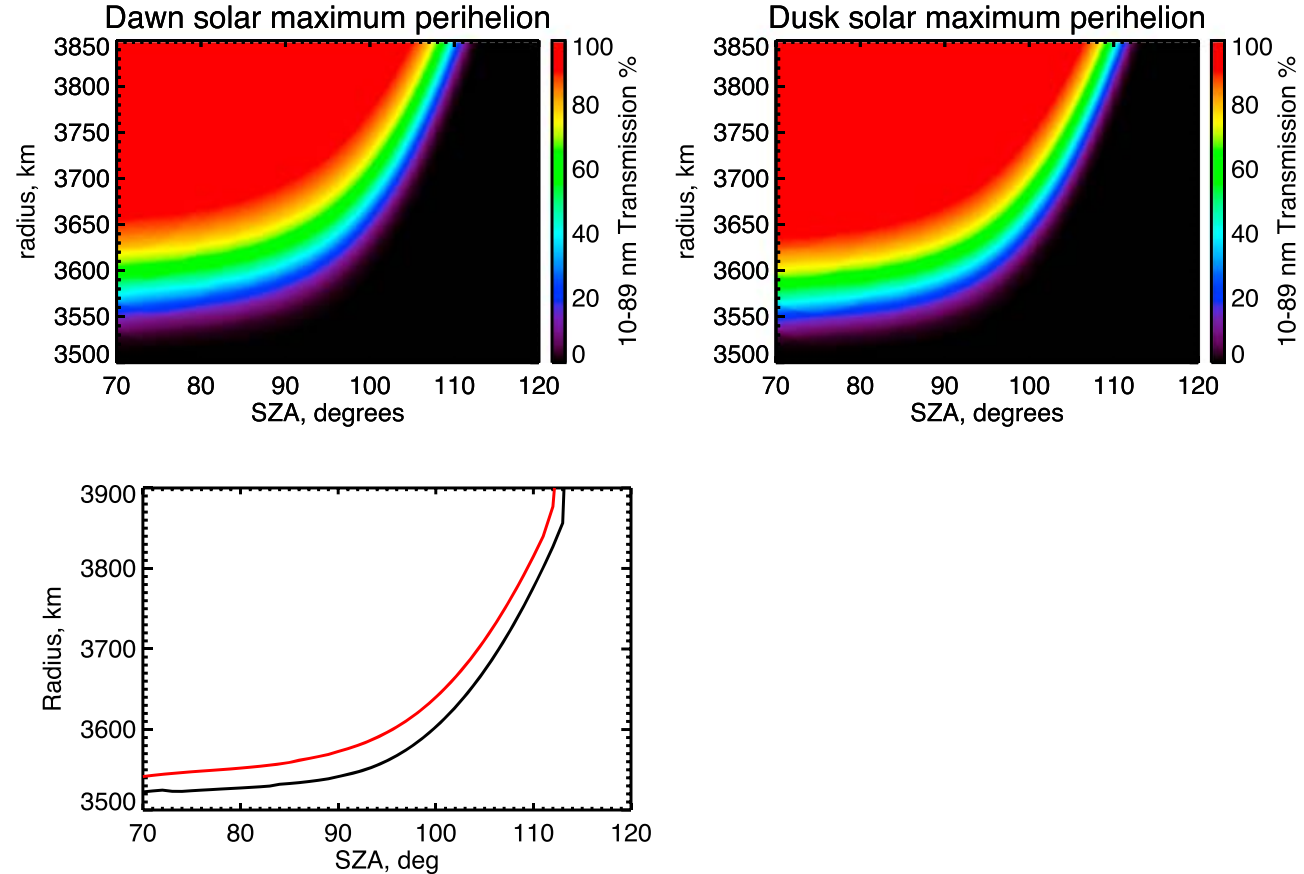

Figure 6. (top) Transmission rate of ionizing EUV photons as a function of the altitude (assuming a mean Martian radius of 3,390 km) and SZA for wavelengths from 10 to $89 \mathrm{~nm}$ for various conditions (local time and seasons). (bottom) The 10\% transmission threshold altitude as a function of SZA, for perihelion dawn (red line) and aphelion dusk (black line) conditions.

threshold leads to a UV terminator distance from 3,548 to $3,587 \mathrm{~km}$, or an altitude from 158.5 to $197.5 \mathrm{~km}$ for a Mars radius of $3,389.5 \mathrm{~km}$.

These values are higher from what we obtained with electron depletions. Several issues have to be kept in mind when determining the UV terminator using electron depletions. First, the choice of the photon transmission threshold induces a small influence: using a lower percentage for the photon transmission 
threshold (below the 5\% limit of electron depletions) may decrease only slightly the altitude of the UV terminator of few kilometers. Besides, we consider that electron depletions are observed when no more photoelectrons are created, without considering the other processes that may bring electrons in the nightside. The boundary delimiting the observation of electron depletions is thus probably at lower altitudes than the effective UV terminator. Another main difference between the two results is that in situ measurements are used to detect electron depletions while the entry used to calculate the transmission rates are taken from a model of the Martian atmosphere. In the end, the interesting point is the large variability of the UV terminator as a function of local time (dawnside or duskside) and seasons, as discussed below.

\section{The Dawn/Dusk Asymmetry}

The UV terminator location is not fixed and homogeneous in time and space. As the other plasma boundaries of the Martian environment, its location depends on several parameters, among which the temperature and density of the neutral atmosphere which have an impact on the absorption of photoelectrons by neutrals. These two parameters both vary with SZA (the Chapman theory (Chapman, 1931a, 1931b) predicts that the density of the atmosphere decrease with SZA) and with seasons. Previous studies showed in particular the presence of significant dawn/dusk ionospheric asymmetries (Benna et al., 2015) that may reveal asymmetries of the background neutral atmosphere (see Fox and Kasprzak (2007) for the Venus case) and thus impact the EUV terminator location.

\subsection{Results Over One Martian Year}

Figure 7 shows the distribution of electron depletions over one Martian year, separating the duskside (local time between 18:00 and 24:00) from the dawnside (local time between 00:00 and 06:00). For the dawnside, the best fit is $126.2 \mathrm{~km}$, with $R^{2}=0.9358$ and a $95 \%$ confidence interval of $(119.5,133 \mathrm{~km})$. For the duskside, the best fit is $126.4 \mathrm{~km}$, with $R^{2}=0.9817$ and the $95 \%$ confidence bounds (123.7, $129.1 \mathrm{~km}$ ). At first glance, no clear asymmetry can be observed between the dawnside and the duskside. However, a closer look to each period will reveal a more variable tendency, which is smoothed over one year.

\subsection{Detail of Each Time Period}

As each period of detection of electron depletions corresponds to a different season, we applied to each of them the same methodology as previously mentioned in section 3.2 to determine the corresponding altitude of the UV terminator, separating the duskside from the dawnside. The results are shown in Figure 8. We mention that the sampling statistics of each altitude/SZA bin (if not at 0 due to orbit coverage) is large enough to provide statistically significant results, with a mean number of passages per bin on average between 30 and 100. The number of depletions is given in each panel, and ranges from 178 only (during the period 3 in the dusk sector) to 85,404 (during the period 2 in the dawn sector).

Looking at Figure 8, the UV terminator is apparently observed at various altitudes above the optical terminator, depending on the period considered:

1. First period: electron depletions have equally been observed on the duskside and on the dawnside. However, few observations have been made at low altitudes and low SZA so that setting a clear value for the altitude of the UV terminator is difficult. Moreover, the largest nightside densities recorded by MAVEN have been observed at high northern altitudes during this time period coincidently with a major solar energetic particle event (Lee et al., 2017). The presence of such events is likely to have changed the altitude of the UV terminator compared to its nominal value.

2. Second period: very few events have been observed on the duskside due to the orbitography of the spacecraft. However, the few events obtained were at low altitudes and low SZA so that their distribution shows a clear boundary at $116 \mathrm{~km}\left(R^{2}=0.9881\right)$. Concerning the dawnside, the fit on the $5 \%$ line was not possible due to a bump of data we cannot explain yet. The fit has then been made on the $10 \%$ line, but the consistency is not as good as the other ones $\left(R^{2}=0.8298\right)$.

3. Third period: very few events have been observed on the duskside due to the orbitography of the spacecraft. Contrary to the second period, these events were located at high altitudes so that the fitting made is not reliable $\left(R^{2}=0.6896\right)$. 

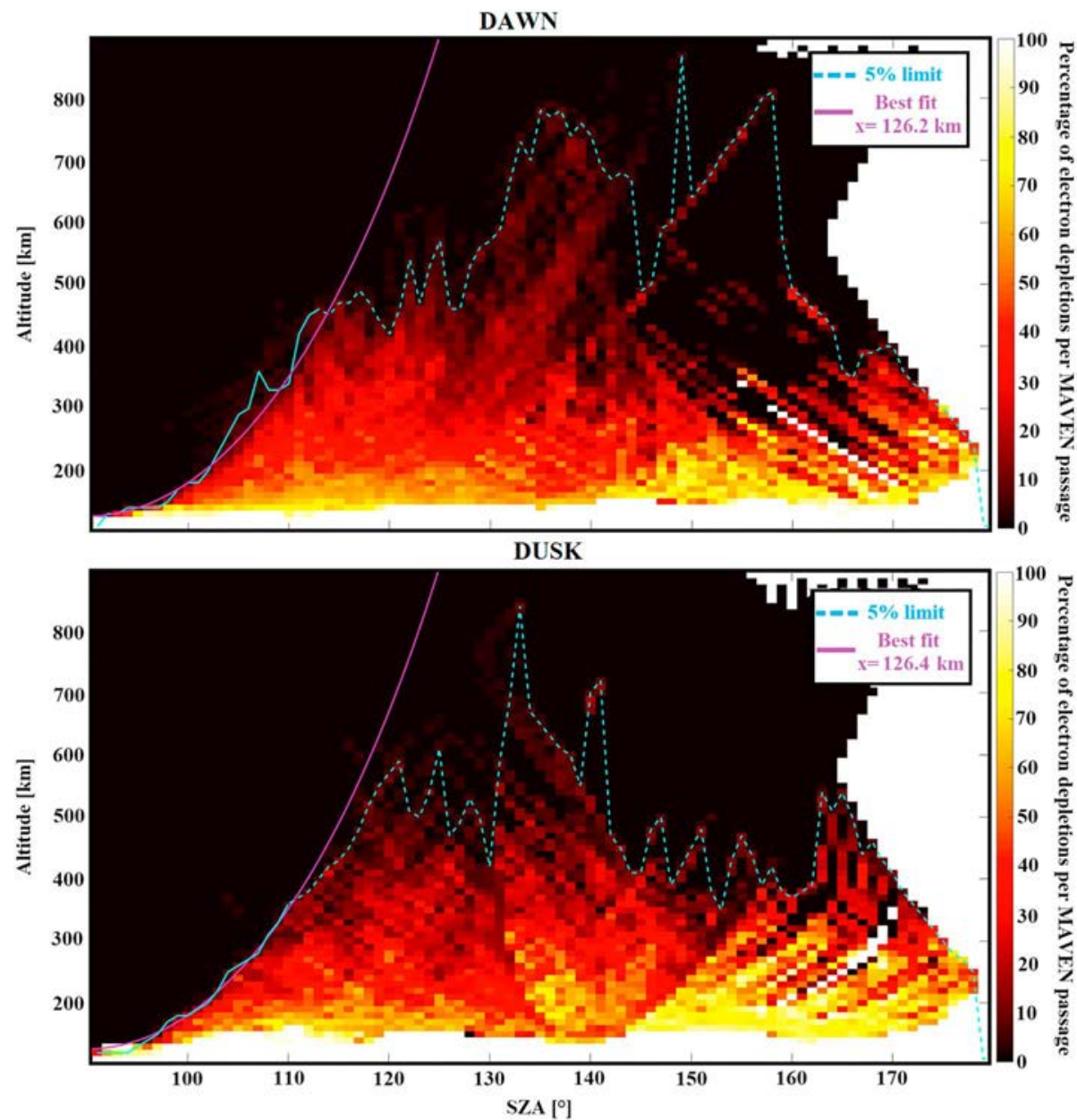

Figure 7. Distribution of the percentage of electron depletions per MAVEN orbit in SZA versus altitude. Data from November 2014 to March 2017 have been taken into account. The areodetic altitude has been used. The top panel corresponds to observations made on the dawnside (corresponding to a total of 270,680 depletion time steps) and the bottom panel corresponds to observation made on the duskside (corresponding to a total of 172,578 depletion time steps).

1. Fourth period: this period has a perfect coverage in both dawnside and duskside. It is the only period allowing to set a clear altitude for the UV terminator on both sides.

2. Fifth period: the removal of spacecraft charging events leads to a low number of depletions on the dawnside. The coverage is thus too limited to provide a UV terminator altitude on the dawnside.

The position of the UV terminator apparently rises or decreases around the average altitude found in the previous section depending on the seasons and between the dawnside and the duskside. However, the MAVEN coverage does not enable us to set a clear position at all periods, the fourth period being the only one with a good enough coverage for dawn and dusk.

\subsection{Focus on the Fourth Period: Comparison With NGIMS Data}

The fourth period turned out to be the only period during which the UV terminator is clearly identifiable both on the duskside and dawnside. The results of our study set the UV terminator at $123.1 \mathrm{~km}$ above the optical terminator in the dawnside $\left(R^{2}=0.9851\right.$ and $95 \%$ confidence bounds of $\left.(118.8,127.5 \mathrm{~km})\right)$, whereas it is at $131 \mathrm{~km}$ in the duskside $\left(R^{2}=0.9873\right.$ and the $95 \%$ confidence bounds $(127.2,135.9 \mathrm{~km})$ ). The dusk terminator is thus above the dawn terminator by $8 \mathrm{~km}$.

The use of the photon transmission model based on the MGCM model results (for the conditions of period 4; i.e., solar average and equinox) as in section 3.4 yields a similar difference of $11 \mathrm{~km}$ between the dawn and dusk UV terminator altitudes. The MGCM model indeed predicts an asymmetry in the composition of the 

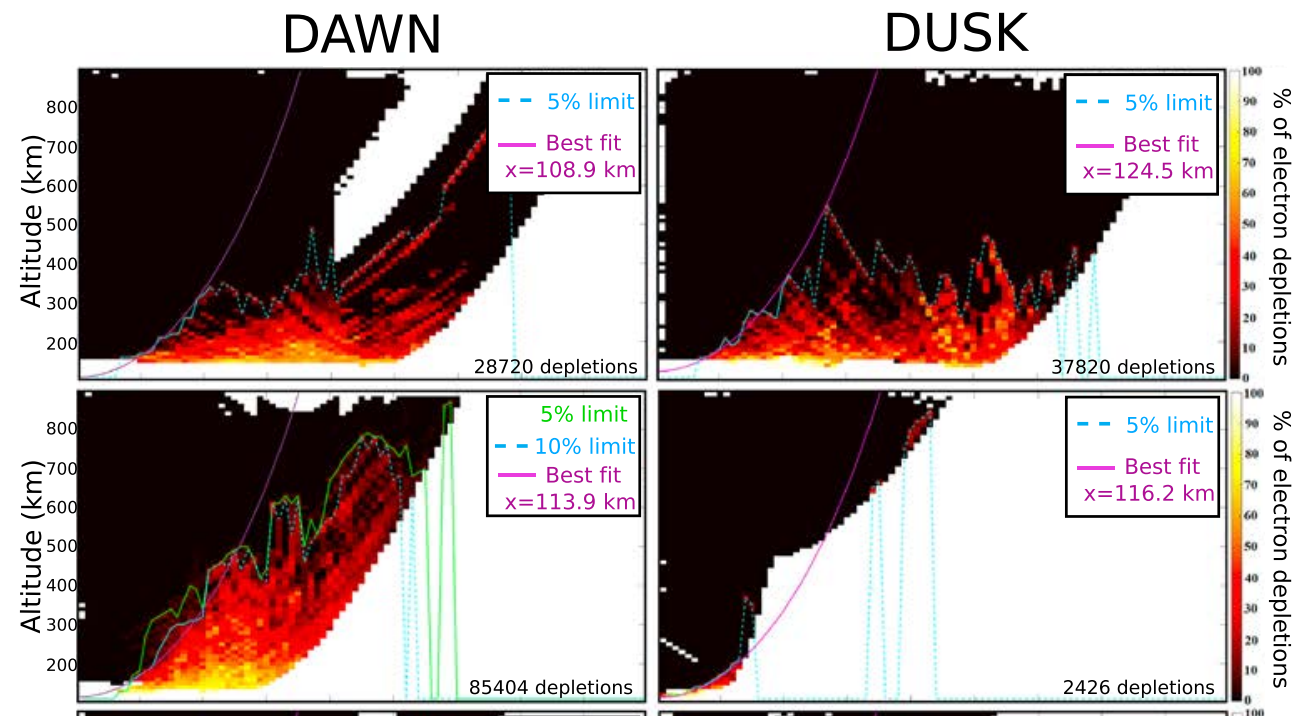

웅
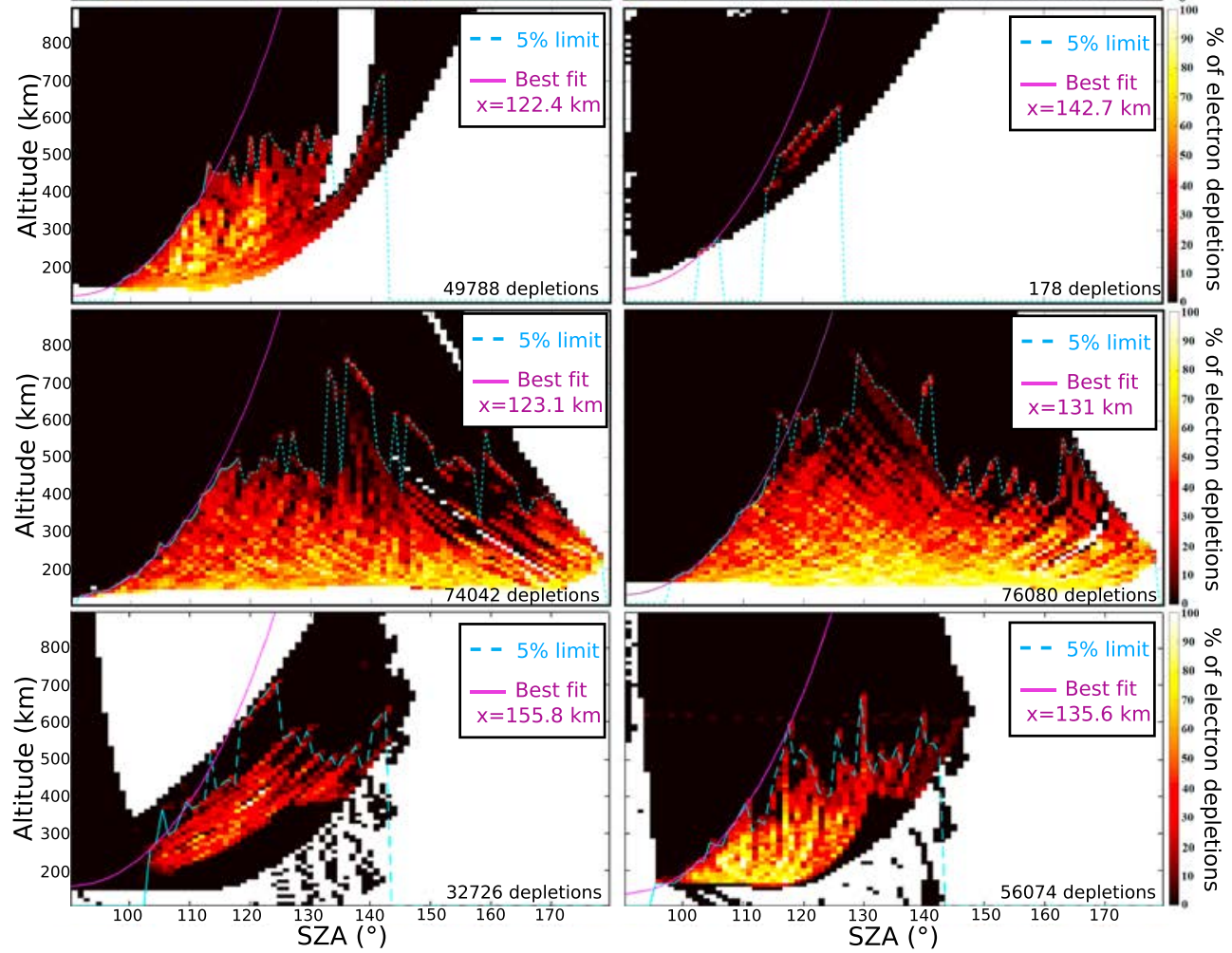

Figure 8. Same as Figure 7 for the five periods during which electron depletions have been detected. The total number of depletion time steps available is shown in each panel.

neutral atmosphere between the duskside and the dawnside driven by cooler temperatures at dawn than at dusk (Chaufray et al., 2014; González-Galindo et al., 2013). Neutrals on the duskside are then expected to have a higher scale height and consequently a greater density at high altitudes than on the dawnside. Photons would consequently be absorbed at higher altitudes in the duskside and the corresponding UV terminator should be located at higher altitudes than in the dawnside.

This model prediction can be checked through neutral density NGIMS measurements. Figure 9 shows the density profiles of the three main neutral species of the neutral atmosphere: $\mathrm{O}, \mathrm{N}_{2}$, and $\mathrm{CO}_{2}$, obtained during the fourth period on the dawnside (black lines) and duskside (red lines). The solid lines correspond to the average NGIMS profiles while the stars correspond to the values from the HELIOSARES models (Leblanc 

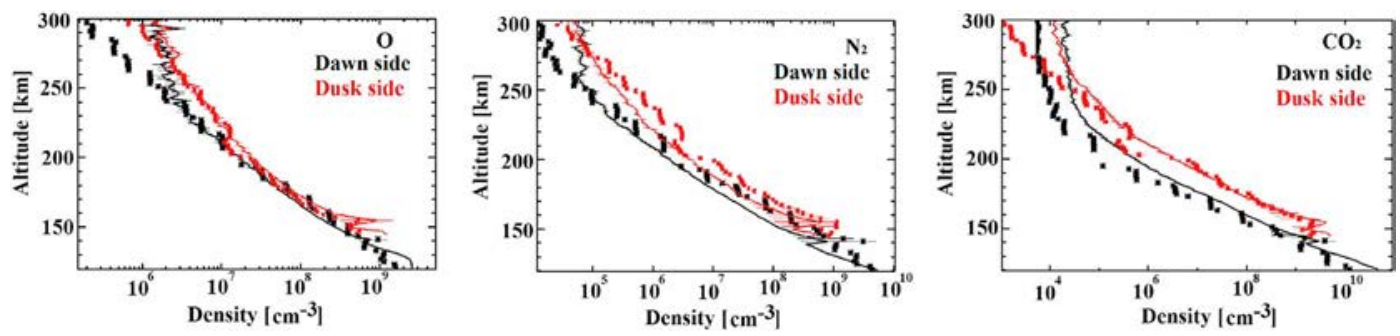

Figure 9. Density profiles of $\mathrm{O}, \mathrm{N}_{2}$, and $\mathrm{CO}_{2}$ obtained by NGIMS during the fourth period. The red lines and stars correspond to the duskside and the black lines and stars correspond to the dawnside. The solid lines are the average NGIMS profiles and the stars correspond to the model set HELIOSARES.

et al., 2017). The HELIOSARES project aims to couple three independent models of the Martian environment: the MGCM, the Mars LATmos Hybrid Simulation magnetospheric model, and the Mars Exospheric Global Model, in order to describe the Martian environment from its surface to the exosphere for any given conditions.

We can see that HELIOSARES values are globally in good consistency with the in situ data, at least for altitudes downward $250 \mathrm{~km}$. For the three species under study, the density on the duskside is actually observed to be higher than on the dawnside, at least for altitudes downward $250 \mathrm{~km}$, which is in agreement with the behavior observed on the distribution of electron depletions.

\section{Discussion on the Seasonal Effect}

It appears interesting to investigate possible seasonal effects of the UV terminator, as an indicator for the atmospheric seasonal variability. However, due to the issues raised in section 4.2 on the coverage of each time period, the comparison of the results obtained with the method using electron depletions during the different seasons is made difficult. Both seasonal and SZA effects are mixed so that no clear conclusion can be set from the data analysis. To observe the seasonal variation of the UV terminator we hence only consider here the results from atmospheric models, which may be validated or not when more data will be available.

\subsection{General Seasonal Effect}

Table 2 bottom line provides the average (over dawn/dusk) modeled UV terminator altitude-from the photon transmission model coupled with the MGCM atmospheric model-depending on the season. One can observe that the UV terminator is thus expected to be the lowest at the aphelion, and the highest at the perihelion, with a variation of $\sim 30 \mathrm{~km}$. One main difference between these two conditions is the UV flux which is higher at the perihelion than at the aphelion. However, the upstream solar flux has little impact on the electron absorption. The parameter that really impacts the electron absorption by the neutral atmosphere is the inclination of the planet on the ecliptic and the induced season due to the differential atmospheric photon absorption according to the latitude. In the hemisphere which is in summer, the temperature is higher, so as the neutral scale height. The neutral density at high altitudes is then higher in the summer hemisphere than in the winter hemisphere, implying an absorption of UV photons at higher altitudes and consequently a higher UV terminator in the summer hemisphere. As the calculations have been made at the equator, this effect is reduced but still significant according to Table 2 results. This seasonal effect is also confirmed by a Monte Carlo radiation transfer model (Toublanc et al., 1995; updated at Titan (Beth et al., 2014; Lebonnois \& Toublanc, 1999)) and adapted to Mars that

Table 2

Altitude of the UV Terminator Modeled Depending on the Season and Local Time Sector (Dawn, Dusk, Average) Considered

\begin{tabular}{lccc}
\hline UV terminator altitude $(\mathrm{km})$ & Perihelion & Equinox & Aphelion \\
\hline Dawn & 197 & 171 & 165 \\
Dusk & 186 & 182 & 158 \\
Average dawn/dusk & 192 & 177 & 162 \\
\hline
\end{tabular}

follows photons in absorption and diffusion in the atmosphere, with very similar results in terms of transmission at different seasons.

Sánchez-Cano et al. (2018) recently investigated the seasonal, latitudinal, and solar cycle variability of the thermosphere-ionosphere coupling by monitoring 10 years of Mars Express total electron content (TEC) observations. They observed in particular that the TEC profile follows the irradiance profile with a maximum near perihelion and a minimum near aphelion. This is fully in agreement with Table 2 model results for the 

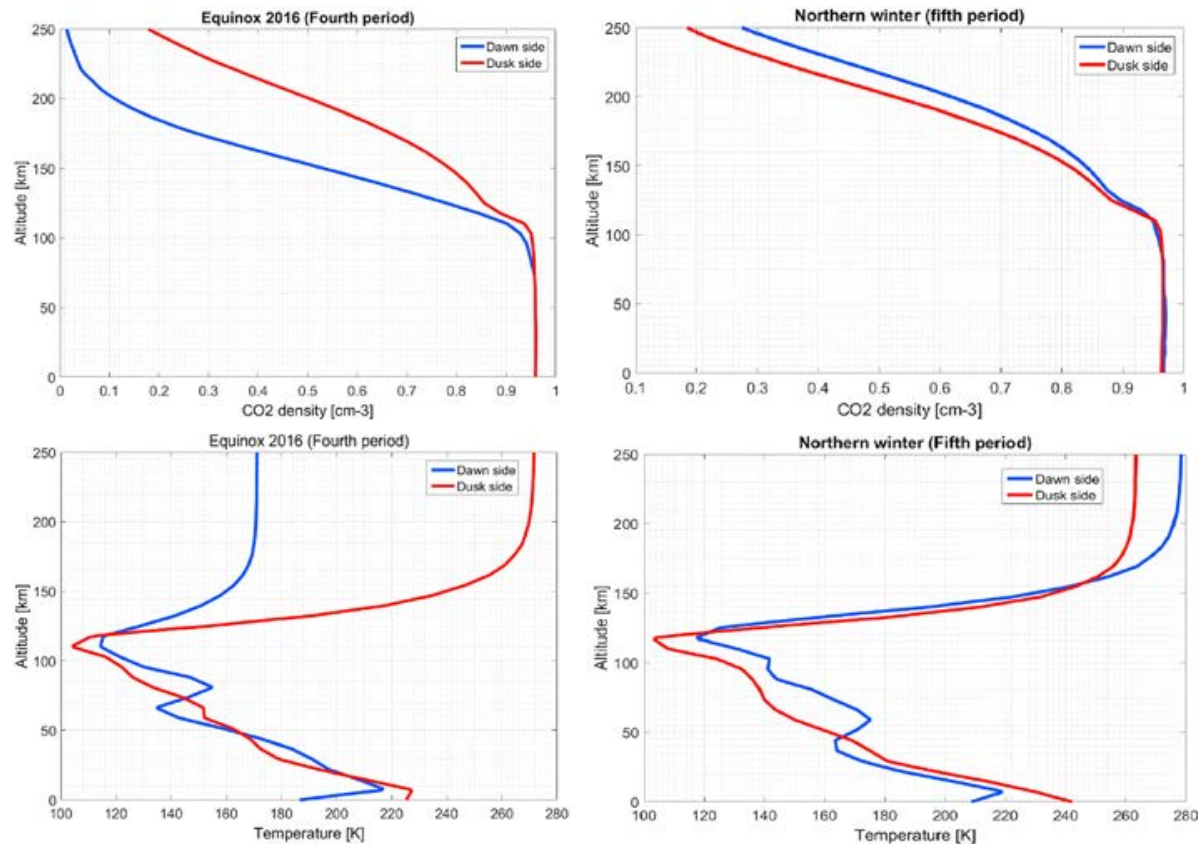

Figure 10. (first row) Density and (second row) temperature profiles on the duskside and dawnside from the MGCM model on 1 August 2016 (i.e., equinox conditions) for the first column and on 22 January 2017 (perihelion conditions) for the second column.

UV terminator altitude, that is maximum at perihelion and minimum at aphelion, due to the same reason: the solar flux is the dominant factor for ionization (and thus for the TEC value) and induces a heating and an inflation of the thermosphere that absorbs more efficiently the photons (and thus enhances the UV terminator altitude). Unfortunately, no further comparison can be made with this study for several reasons: the TEC value could essentially be obtained in the dusk sector and not in the dawn sector due to the Mars-Express orbit evolution, and our method (based on large periods mapping of the suprathermal electron depletions) combined with the limited altitude-SZA coverage of MAVEN at some periods does not allow us to monitor the UV terminator altitude along all the seasons.

\subsection{Seasonal Variation of the Dawn/Dusk Asymmetry}

We saw in the previous section that, at the equinox, the dusk terminator is higher than the dawn terminator, which is consistent with models as well as neutral observations. However, Table 2 model results (transmission model by R. Lillis combined with MGCM neutral profiles) predict that this configuration is specific to the equinox: the dawn/dusk UV terminator asymmetry reverses at the perihelion and aphelion of Mars, whereas this asymmetry is expected to be driven by the cooler temperatures at dawn compared to dusk.

In order to check if this inversion is a photon transmission model issue, we compared these results with those obtained with the radiative transfer model of D. Toublanc (adapted to Mars from Toublanc et al. (1995)). In order to observe the inversion between seasons, we used two sets of input conditions from the MGCM (pressure, temperature) corresponding to the fourth (equinox conditions) and fifth (perihelion conditions) periods of detection of electron depletions. The results (not shown) are the same: the dusk terminator is higher than the dawn terminator at the equinox and the asymmetry reverses at the perihelion of Mars.

The dawn/dusk asymmetry reversal thus probably originates from the input neutral profiles used and provided by the MGCM model. We plot in Figure 10 the temperature and pressure profiles typical for both the transmission profiles in Figure 6 and Table 2 results (one for perihelion, one for equinox). One can indeed see an inversion of the density and temperature profiles between the two periods. At the equinox the temperature and the total density are higher on the duskside than on the dawnside, while at perihelion (northern winter) the density is slightly higher in the dawnside and the temperature profile in the dawnside is more complicated. What catches the eyes is the dawn density at the equinox which falls at high altitudes 
compared to what is observed at the northern winter and compared to the dusk profile, probably induced by the similar fall-off of the temperature at the same period. The origin of this strong cooling in the dawn sector at equinox is, to our knowledge, unexplained. It could however be related to the strong seasonal variability of the water vapor in Martian atmosphere, whose column density is minimum at equinox (Smith, 2002) and whose amount plays a key role in the atmospheric cooling at Mars. More precisely, if we run MGCM simulations to analyze the seasonal variation of the water vapor volumic mixing ratio, we can show that it has a very similar behavior to the temperature profile above $100-\mathrm{km}$ altitude, with more water vapor on the duskside than on the dawnside at equinox, and a reverse situation at perihelion. Whether this similarity is a consequence or a reason remains however to be studied in future work.

\section{Conclusions}

Suprathermal electron depletions are structures of the nightside ionosphere of Mars resulting from an equilibrium between electron source and loss processes. Since on Mars the main ionization process is photoionization of the neutral atmosphere by UV and EUV photons, electron depletions are in first approximation only observed below the UV terminator, thus providing a new and indirect method to set its localization.

On average over one Martian year, the UV terminator has been found to be located $123 \mathrm{~km}$ above the optical terminator. The UV terminator has been observed to lay as expected at higher altitudes on the duskside than on the dawnside at the equinox, in agreement with atmospheric models and recent results from NGIMS: the warmer dusk conditions lead to an inflation of the atmosphere that absorbs more efficiently the photons, and thus enhances the UV terminator altitude. However, atmospheric models predict that the situation reverses at the perihelion and the aphelion, but data did not enable us to confirm or invalidate this unexplained inversion yet. The use of the fifth period of detection of electron depletions, which equally covers the duskside and dawnside, could have enabled to support the models and to observe the inversion of the location of the dawn and dusk UV terminator, but the removal of spacecraft charging events prevents from setting a clear conclusion. If confirmed by future analysis of electron suprathermal depletions at later periods, this inversion would probably be induced by a very strong cooling of the thermosphere at dawn versus dusk at equinox, possibly related to the seasonal behavior of water vapor in the Martian atmosphere.

The analysis of the suprathermal electron depletions thus not only gives information on the plasma dynamics and magnetic field topology (Steckiewicz et al., 2015, 2016) but may also provide insight into the seasonal and local time variability of the Martian thermosphere.

\section{Acknowledgments}

This work has been supported by the French space agency CNES for the par based on observations obtained with the SWEA instrument on MAVEN. The MAVEN project is supported by NASA through the Mars Exploration Program. The authors acknowledge the support of the MAVEN project and particularly of the instrument and science teams. Data analysis was performed with the AMDA science analysis system (http:// amda.cdpp.eu/) provided by the Centre de Données de la Physique des Plasmas (CDPP) supported by CNRS, CNES, Observatoire de Paris, and Université Paul Sabatier, Toulouse, France. The MAVEN data used in this paper are publicly available through the Planetary Data System (http://ppi.pds. nasa.gov/).

\section{References}

Acuña, M. H., Connerney, J. E. P., Wasilewski, P., Lin, R. P., Anderson, K. A., Carlson, C. W., et al. (1998). Magnetic field and plasma observations at Mars: Initial results of the Mars Global Surveyor mission. Science, 279(5357), 1676-1680. https://doi.org/10.1126/ science.279.5357.1676

Andersson, L., Ergun, R. E., Delory, G. T., Eriksson, A. I., Westfall, J., Reed, H., et al. (2015). The Langmuir Probe and Waves instrument for MAVEN. Space Science Reviews, 195(1-4), 173-198. https://doi.org/10.1007/s11214-015-0194-3

Benna, M., Mahaffy, P. R., Grebowsky, J. M., Fox, J. L., Yelle, R. V., \& Jakosky, B. M. (2015). First measurements of composition and dynamics of the Martian ionosphere by MAVEN's Neutral Gas and Ion Mass Spectrometer. Geophysical Research Letters, 42, 8958-8965. https://doi.org/10.1002/2015GL066146

Beth, A., Garnier, P., Toublanc, D., Dandouras, I., Mazelle, C., \& Kotova, A. (2014). Modeling the satellite particle population in the planetary exospheres: Application to Earth, Titan and Mars. Icarus, 227, 21-36. https://doi.org/10.1016/j.icarus.2013.07.031

Bougher, S., Jakosky, B., Halekas, J., Grebowsky, J., Luhmann, J., Mahaffy, P., et al. (2015). Early MAVEN deep dip campaigns: First results and implications. Sciences, 350(6261), aad0459. https://doi.org/10.1126/science.aad0459

Chapman, S. (1931a). The absorption and dissociative or ionizing effect of monochromatic radiation in an atmosphere on a rotating Earth Proceedings of the Physical Society, 43(1), 26-45. https://doi.org/10.1088/0959-5309/43/1/305

Chapman, S. (1931b). The absorption and dissociative or ionizing effect of monochromatic radiation in an atmosphere on a rotating Earth: Part II-Grazing incidence. Proceedings of the Physical Society, 43(5), 483-501. https://doi.org/10.1088/0959-5309/43/5/302

Chaufray, J.-Y., Gonzalez-Galindo, F., Forget, F., Lopez-Valverde, M., Leblanc, F., Modolo, R., et al. (2014). Three-dimensional Martian ionosphere model: II. Effect of transport processes due to pressure gradients. Journal of Geophysical Research: Planets, 119, $1614-1636$. https://doi.org/10.1002/2013JE004551

Connerney, J. E. P., Espley, J., Lawton, P., Murphy, S., Odom, J., Oliverson, R., \& Sheppard, D. (2015). The MAVEN magnetic field investigation. Space Science Reviews, 195(1-4), 257-291. https://doi.org/10.1007/s11214-015-0169-4

Cui, J., Galand, M., Coates, A. J., Zhang, T. L., \& Müller-Wodarg, I. C. F. (2011). Suprathermal electron spectra in the Venus ionosphere. Journal of Geophysical Research, 116, A04321. https://doi.org/10.1029/2010JA016153

Eparvier, F., Chamberlin, P. C., \& Woods, T. N. (2015). The Solar Extreme Ultraviolet Monitor for MAVEN. Space Science Reviews, 195(1-4), 293-301. https://doi.org/10.1007/s11214-015-0195-2 
Fillingim, M. O., Peticolas, L. M., Lillis, R. J., Brain, D. A., Halekas, J. S., Lummerzheim, D., \& Bougher, S. W. (2010). Localized ionization patches in the nighttime ionosphere of Mars and their electrodynamic consequences. Icarus, 206(1), 112-119. https://doi.org/10.1016/j. icarus.2009.03.005

Forget, F., Hourdin, F., Fournier, R., Hourdin, C., Talagrand, O., Collins, M., et al. (1999). Improved general circulation models of the Martian atmosphere from the surface to above $80 \mathrm{~km}$. Journal of Geophysical Research, 104(E10), 24,155-24,175. https://doi.org/10.1029/ 1999JE001025

Fox, J. L., \& Kasprzak, W. T. (2007). Near-terminator Venus ionosphere: Evidence for a dawn/dusk asymmetry in the thermosphere. Journal of Geophysical Research, 112, E09008. https://doi.org/10.1029/2007JE002899

González-Galindo, F., Chaufray, J.-Y., López-Valverde, M. A., Gilli, G., Forget, F., Leblanc, F., et al. (2013). Three-dimensional Martian ionosphere model: I. The photochemical ionosphere below $180 \mathrm{~km}$. Journal of Geophysical Research: Planets, 118, 2105-2123. https:// doi.org/10.1002/jgre.20150

González-Galindo, F., Forget, F., López-Valverde, M. A., Angelats i Coll, M., \& Millour, E. (2009). A ground-to-exosphere Martian general circulation model: 1. Seasonal, diurnal, and solar cycle variation of thermospheric temperatures. Journal of Geophysical Research, 114, E04001. https://doi.org/10.1029/2008JE003246

Hall, B. E. S., Lester, M., Nichols, J. D., Snchez-Cano, B., Andrews, D. J., Opgenoorth, H. J., \& Frnz, M. (2016). A survey of superthermal electron flux depressions, or electron holes, within the illuminated Martian induced magnetosphere. Journal of Geophysical Research: Space Physics, 121, 4835-4857. https://doi.org/10.1002/2015JA021866

Huebner, W. F., \& Mukherjee, J. (2015). Photoionization and photodissociation rates in solar and blackbody radiation fields. Planetary and Space Science, 106, 11-45. https://doi.org/10.1016/j.pss.2014.11.022

Jakosky, B. M., Lin, R. P., Grebowsky, J. M., Luhmann, J. G., Mitchell, D. F., Beutelschies, G., et al. (2015). The Mars Atmosphere and Volatile Evolution (MAVEN) mission. Space Science Reviews, 195(1-4), 3-48. https://doi.org/10.1007/s11214-015-0139-x

Kivelson, M. G., \& Russel, C. T. (1995). In M. G. Kivelson, \& C. T. Russell (Eds.), Introduction to space physics. Cambridge, UK: Cambridge University Press. ISBN: 0521451043.

Leblanc, F., Chaufray, J. Y., Modolo, R., Leclercq, L., Curry, S., Luhmann, J., et al. (2017). On the origins of Mars' exospheric nonthermal oxygen component as observed by MAVEN and modeled by HELIOSARES. Journal of Geophysical Research: Planets, 122, $2401-2428$. https://doi.org/10.1002/2017JE005336

Lebonnois, S., \& Toublanc, D. (1999). Actinic fluxes in Titan's atmosphere, from one to three dimensions: Application to high-latitude composition. Journal of Geophysical Research, 104(E9), 22,025-22,034. https://doi.org/10.1029/1999JE001056

Lee, C., Hara, T., Halekas, J. S., Thiemann, E., Chamberlin, P., Eparvier, F., et al. (2017). MAVEN observations of the solar cycle 24 space weather conditions at Mars. Journal of Geophysical Research: Space Physics, 122, 2768-2794. https://doi.org/10.1002/ 2016JA023495

Lillis, R. J., \& Brain, D. A. (2013). Nightside electron precipitation at Mars: Geographic variability and dependence on solar wind conditions. Journal of Geophysical Research: Space Physics, 118, 3546-3556. https://doi.org/10.1002/jgra.50171

Lillis, R. J., Fillingim, M. O., \& Brain, D. A. (2011). Three-dimensional structure of the Martian nightside ionosphere: Predicted rates of impact ionization from Mars Global Surveyor magnetometer and Electron Reflectometer measurements of precipitating electrons. Journal of Geophysical Research, 116, A12317. https://doi.org/10.1029/2011JA016982

Lillis, R. J., Mitchell, D. L., Lin, R. P., \& Acuña, M. H. (2008). Electron reflectrometry in the Martian atmosphere. Icarus, 194(2), 544-561. https://doi.org/10.1016/j.icarus.2007.09.030

Mahaffy, P. R., Benna, M., King, T., Harpold, D. N., Arvey, R., Barciniak, M., et al. (2015). The Neutral Gas and Ion Mass Spectrometer on the Mars Atmosphere and Volatile Evolution Mission. Space Science Reviews, 195(1-4), 49-73. https://doi.org/10.1007/s11214-0140091-1

Mantas, G. P., \& Hanson, W. B. (1979). Photoelectron fluxes in the Martian ionosphere. Journal of Geophysical Research, 84(A2), 369-385. https://doi.org/10.1029/JA084iA02p00369

Mitchell, D. L., Lin, R. P., Mazelle, C., Rème, H., Cloutier, P. A., Connerney, J. E. P., et al. (2001). Probing Mars' crustal magnetic field and ionosphere with the MGS Electron Reflectometer. Journal of Geophysical Research, 106(E10), 23,419-23,427. https://doi.org/10.1029/ 2000JE001435

Mitchell, D. L., Lin, R. P., Rème, H., Crider, D. H., Cloutier, P. A., Connerney, J. E. P., et al. (2000). Oxygen Auger electrons observed in Mars' ionosphere. Geophysical Research Letters, 27(13), 1871-1874. https://doi.org/10.1029/1999GL010754

Mitchell, D. L., Mazelle, C., Sauvaud, J. A., Thocaven, J. J., Rouzaud, J., Fedorov, A., et al. (2016). The MAVEN Solar Wind Electron Analyzer. Space Science Reviews, 200(1-4), 495-528. https://doi.org/10.1007/s11214-015-0232-1

Morschhauser, A., Lesur, V., \& Grott, M. (2014). A spherical harmonic model of the lithospheric magnetic field of Mars. Journal of Geophysical Research: Planets, 119, 1162-1188. https://doi.org/10.1002/2013JE004555

Sakai, S. (2016). Electron energetics in the Martian dayside ionosphere: Model comparisons with MAVEN data. Journal of Geophysical Research: Space Physics, 121, 7049-7066. https://doi.org/10.1002/2016JA022782

Sánchez-Cano, B., Lester, M., Witasse, O., Blelly, P. L., Indurain, M., Cartacci, M., et al. (2018). Spatial, seasonal, and solar cycle variations of the Martian total electron content (TEC): Is the TEC a good tracer for atmospheric cycles? Journal of Geophysical Research: Planets, 123, 1746-1759. https://doi.org/10.1029/2018JE005626

Shane, A. D., Xu, S., Liemohn, M. W., \& Mitchell, D. L. (2016). Mars nightside electrons over strong crustal fields. Journal of Geophysical Research: Space Physics, 121, 3808-3823. https://doi.org/10.1002/2015JA021947

Smith, M. D. (2002). The annual cycle of water vapor as observed by the Thermal Emission Spectrometer. Journal of Geophysical Research, 107(E11), 5115. https://doi.org/10.1029/2001JE001522

Steckiewicz, M., Garnier, P., André, N., Mitchell, D. L., Andersson, L., Penou, E., et al. (2016). Comparative study of the Martian suprathermal electron depletions based on Mars Global Surveyor, Mars Express and the Mars Atmosphere and Volatile Evolution mission observations. Journal of Geophysical Research: Space Physics, 122, 857-873. https://doi.org/10.1002/2016JA023205

Steckiewicz, M., Mazelle, C., Garnier, P., André, N., Penou, E., Beth, A., et al. (2015). Altitude dependence of nightside Martian suprathermal electron depletions as revealed by MAVEN observations. Geophysical Research Letters, 42, 8877-8884. https://doi.org/ $10.1002 / 2015$ GL065257

Thiemann, E. M. B., Chamberlin, P. C., Eparvier, F. G., Templeman, B., Woods, T. N., Bougher, S. W., \& Jakosky, B. M. (2017). The MAVEN EUVM model of solar spectral irradiance variability at Mars: Algorithms and results. Journal of Geophysical Research: Space Physics, 122 , 2748-2767. https://doi.org/10.1002/2016JA023512

Toublanc, D., Parisot, J. P., Brillet, J., Gautier, D., Raulin, F., \& McKay, C. P. (1995). Photochemical modeling of Titan's atmosphere. Icarus, 113(1), 2-26. https://doi.org/10.1006/icar.1995.1002 
Venot, O., Bénilan, Y., Fray, N., Gazeau, M. C., Lefèvre, F., Es-sebbar, E., et al. (2018). VUV-absorption cross section of carbon dioxide from 150 to $800 \mathrm{~K}$ and applications to warm exoplanetary atmospheres. Astronomy \& Astrophysics, 609, A34. https://doi.org/10.1051/0004$6361 / 201731295$

Xu, S., Liemohn, M., Bougher, S., \& Mitchell, D. (2016). Martian high-altitude photoelectrons independent of solar zenith angle. Journal of Geophysical Research: Space Physics, 121, 3767-3780. https://doi.org/10.1002/2015JA022149

Xu, S., Mitchell, D., Liemohn, M., Fang, X., Ma, Y., Luhmann, J., et al. (2017). Martian low-altitude magnetic topology deduced from MAVEN/SWEA observations. Journal of Geophysical Research: Space Physics, 122, 1831-1852. https://doi.org/10.1002/ 2016JA023467 